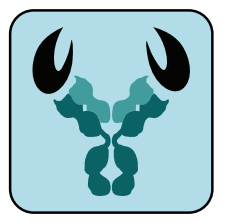

Journal of Experimental \& Clinical Cancer Research

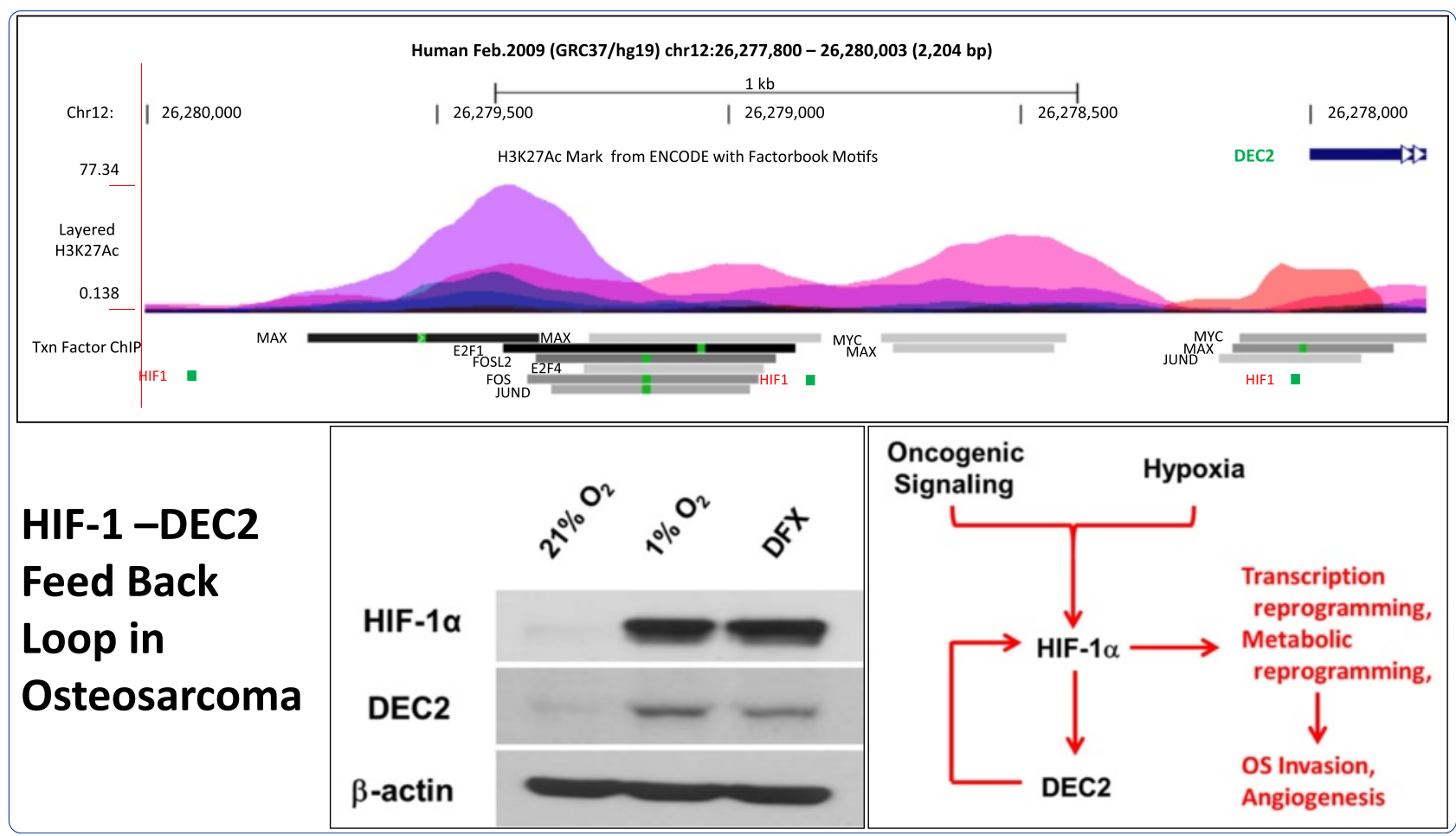

\title{
DEC2 expression is positively correlated with HIF-1 activation and the invasiveness of human osteosarcomas
}

Hu et al. 


\title{
DEC2 expression is positively correlated with HIF-1 activation and the invasiveness of human osteosarcomas
}

Tu Hu ${ }^{1 \dagger}$, Nengbin He ${ }^{1 \dagger}$, Yunsong Yang ${ }^{2}$, Chengqian Yin ${ }^{3}$, Nianli Sang ${ }^{3,4^{*}}$ and Qingcheng Yang ${ }^{1 *}$

\begin{abstract}
Background: Osteosarcoma is the most common malignancy of bone. HIF-1 (hypoxia-inducible factor 1) activation is critical for the metabolic reprogramming and progression of solid tumors, and DEC2 (differentiated embryonic chondrocyte gene 2) has been recently reported to suppress HIF-1 in human breast and endometrial cancers. However, the roles of HIF-1 and DEC2 in human osteosarcomas remain unclear.

Methods: We evaluated the correlation of DEC2 and HIF-1 expression to the prognosis, and studied the roles of DEC2 and HIF-1 activation in the invasiveness of osteosarcoma. Multiple approaches including immunohistochemical staining of clinical osteosarcoma tissues, siRNA-based knockdown and other molecular biology techniques were used. Particularly, by using a repetitive trans-well culture-based in vitro evolution system, we selected a more invasive subpopulation (U2OS-M) of osteosarcoma cells from U2OS and used it as a model to study the roles of DEC2 and HIF-1 in the invasiveness of osteosarcoma.

Results: We found that the expression of DEC2 was positively correlated with HIF-1a levels, and HIF-1a expression positively correlated with poor prognosis in osteosarcomas. DEC2 knockdown in osteosarcoma cell lines (U2OS, MNNG and 143B) attenuated HIF-1a accumulation and impaired the up-regulation of HIF-1 target genes in response to hypoxia. Compared with the low invasive parental U2OS, U2OS-M showed higher levels of DEC2 expression which were confirmed at both mRNA and protein levels. Importantly, we found that the increased DEC2 expression resulted in a more rapid accumulation of HIF-1a in U2OS-M cells in response to hypoxia. Finally, we found that HIF-1 activation is sufficient to upregulate DEC2 expression in osteosarcoma cells.

Conclusion: Taken together, whereas DEC2 was found to promote HIF-1a degradation in other types of tumors, our data indicate that DEC2 facilitates HIF-1a stabilization and promotes HIF-1 activation in osteosarcoma. This implies that DEC2 may contribute to the progression and metastasis of human osteosarcoma by sensitizing tumor cells to hypoxia. On the other hand, HIF-1 activation may contribute to the expression of DEC2 in osteosarcoma. This is the first demonstration of a novel DEC2-HIF-1 vicious cycle in osteosarcoma and a tumor-type specific role for DEC2.
\end{abstract}

Keywords: Osteosarcoma, DEC2, SHARP1, HIF-1a, Metastasis, Prognosis

\footnotetext{
*Correspondence: nianli.sang@drexel.edu; tjyqc@163.com

${ }^{\dagger}$ Equal contributors

${ }^{3}$ Departments of Biology, Pathology \& Laboratory Medicine, Drexel University

CoAS, 3245 Chestnut St, PISB 417, Philadelphia, PA 19104, USA

'Department of Orthopedics, Shanghai Jiao Tong University Affiliated Sixth

People's Hospital, No.600, Yishan Road, Shanghai 200233, China

Full list of author information is available at the end of the article
} 


\section{Background}

Osteosarcoma is the most common malignancy of bone, with a peak incidence in the second and third decades of life [1]. Osteosarcoma is very aggressive and has a high tendency to metastasis [2]. The 5-year event-free survival rate (EFS) for patients diagnosed with localized disease has improved since the invention of preoperative and postoperative chemotherapy. However, the prognosis for patients with metastatic osteosarcoma remains poor with a 5 -year EFS of no more than 20\% [3]. Currently, prognostic evaluation of osteosarcoma is mainly based on clinical parameters while useful molecular markers to predict tumor aggressiveness and to guide clinical treatment need to be explored [4].

The activation of hypoxia-inducible factor-1 (HIF-1) has been observed in osteosarcoma [5]; and clinically, osteosarcoma is commonly associated with abundant blood supply resulting from angiogenesis. Like other rapid growing solid tumors, osteosarcoma may develop a spatiotemporal hypoxic microenvironment during progression, hence activating HIF-1. HIF-1 is a heterodimeric transcription factor formed by HIF- $1 \alpha$ and HIF- $1 \beta$ (also known as aryl hydrocarbon receptor nuclear translocator 1, ARNT1). HIF-1 is the main regulator of tumor angiogenesis, and regulates the expression of many genes relevant to oxygen transport, glucose metabolism, cell proliferation and apoptosis [6-8]. The protein level of HIF $-1 \alpha$ is regulated by oxygen concentrations while HIF- $1 \beta$ is constitutively expressed. In addition to hypoxic activation of HIF-1 $\alpha$ transactivation activity by inhibiting hydroxylation of the transactivation domain $[9,10]$, hypoxic stabilization of HIF- $1 \alpha$ is the other important mechanism for functional activation of HIF-1. Under normoxic conditions, oxygen-dependent hydroxylation of two prolyl residues of HIF- $1 \alpha$ triggers a VHL (von Hippel Lindau)-mediated ubiquitination and proteasomal degradation of HIF-1 $\alpha$ [6]. Hypoxia suppresses prolyl hydroxylase activity, preventing HIF-1 $\alpha$ from hydroxylation and subsequent ubiquitination, thus stabilizing HIF-1 $\alpha$. Recent studies show that MDM2 might serve as an E3 ligase, facilitating VHL-independent proteasome-dependent degradation of HIF-1 $\alpha$ [11,12]. Moreover, oncogenic signaling caused by either activation of oncogenes or loss of tumor suppressor functions may provide additional mechanisms to activate HIF-1 $[6,13]$. Increased HIF-1 $\alpha$ levels have been found in many tumor types, accompanied by increased expression of HIF-1 target genes, including but not limited to, vascular endothelial growth factor A (VEGFA), phosphoglycerate kinase 1 (PGK1), angiopoietin-like 4 (ANGPTL4), carbonic anhydrase IX (CAIX) and hexose kinase 2 (HK2) [14]. HIF-1 $\alpha$ overexpression has been correlated with high risk of metastasis and high mortality in many human cancers, including breast cancer and renal cell carcinoma [15-17], whereas the molecular mechanism underlying its overexpression in osteosarcoma and potential impact on osteosarcoma progression have not been fully understood.

Differentiated embryonic chondrocyte gene 2 (DEC2, also known as SHARP1 and bHLHE41) is a basic helix-loophelix (bHLH) transcription regulator which has been implicated in regulating cell proliferation, apoptosis and circadian rhythms [18-22]. Recent studies have suggested that DEC2 may regulate HIF-1 $\alpha$ expression in human breast and endometrial cancers [22-25]. In these reports, DEC2 was found to physically interact with and promote HIF- $1 \alpha$ degradation, contributing to its suppressive effects on tumor progression and metastasis. However, it remains unknown whether, and how DEC2 affects HIF-1 activation in osteosarcomas.

In this study, we determined the expression levels of DEC2 and HIF-1 $\alpha$ in osteosarcoma samples and analyzed the correlation between DEC2 expression and HIF- $1 \alpha$ levels. We demonstrated that DEC2 knockdown in U2OS, MNNG and 143B cells led to down-regulation of HIF-1 $\alpha$ protein levels and suppressed HIF-1-dependent activation of downstream target genes. By using an in vitro evolution model, we selected a highly invasive subpopulation (U2OS-M) from U2OS cells, and found that the highly invasive subpopulation had increased expression of DEC2 at both mRNA and protein levels, accompanied by accelerated HIF-1 $\alpha$ accumulation upon hypoxia. Finally, we show that HIF-1 activation is sufficient to enhance DEC2 expression. Taken together, our data suggest that DEC2, which was shown to promote HIF-1 $\alpha$ degradation in other tumors, may facilitate HIF-1 activation and metabolic reprograming in osteosarcomas, and that HIF-1 activation may, in turn, promote DEC2 expression, forming a vicious cycle.

\section{Materials and methods}

\section{Human osteosarcoma samples}

A total of 50 patients treated between 2006 and 2011 at the Department of Orthopedics, Shanghai Jiao Tong University Affiliated Sixth People's Hospital (Shanghai, China) that were followed for 3 years were included in this study. All samples of human osteosarcoma were collected at the time of surgery. The study was approved by the Ethics Committee of Shanghai Jiao Tong University, and informed consent was obtained from all patients included in this study.

\section{Cell lines and cell culture}

The MNNG and U2OS cell lines were purchased from the ATCC repository. 143B was a gift from Dr. M. King (Sydney Kimmel Cancer Center, Philadelphia) [26]. The cells were cultured in Dulbecco's Modified Eagle Medium (DMEM) supplemented with $10 \%$ fetal bovine serum (FBS) (Biowest, South America Origin), $100 \mathrm{U} / \mathrm{ml}$ penicillin 
(Sigma-Aldrich) and $100 \mu \mathrm{g} / \mathrm{ml}$ streptomycin (SigmaAldrich) at $37^{\circ} \mathrm{C}$ in $5 \% \mathrm{CO}_{2}$. The cells were regularly monitored to ensure that they were free of mycoplasma contamination. For hypoxic treatment, the cells were exposed to $1 \% \mathrm{O}_{2}$ with $5 \% \mathrm{CO}_{2}$ at $37^{\circ} \mathrm{C}$ for a duration indicated in each experiment with hypoxia chamber, or hypoxia Workstation (InVIVO2).

\section{Isolation of invasive subpopulation with trans-well chambers}

The trans-well culture was performed as previously reported [27,28]. Briefly, 24-well plate inserts with 8-mm pore size chambers (Corning, USA) were used to isolate highly invasive subpopulation from the cultured U2OS parental cell line. First, cells were suspended in serum-free DMEM to a final cell density of $5 \times 10^{5}$ cells $/ \mathrm{ml}$. $200 \mu \mathrm{l}$ of cell suspension were seeded into the top chamber, which was coated with Matrigel. In the lower chamber, $800 \mu \mathrm{l}$ of DMEM supplemented with $10 \%$ fetal bovine serum was added. Following incubation for $24 \mathrm{~h}$ at $37^{\circ} \mathrm{C}$, the invasive cells on the underside of the membrane were expanded and used for subsequent rounds of selection. After six rounds of selection, the cell subpopulation able to migrate through the membranes was designated as U2OS-M.

\section{Synthetic small interfering RNAs (siRNAs) and RNA interference}

The siRNA specifically targeting DEC2 (siDEC2) and negative control siRNA (siControl) were designed and synthesized by Biotend (Shanghai, China). The sequence used for siDEC2 was: 5'-ACGACACCAAGGAUACCU AdTdT/UAGGUAUCCUUGGUGUCGUdTdT-3' . Cells were transfected with siRNA using Lipofectamine 2000 (Invitrogen) by following the manufacturer's protocol. For RNA extraction and western blotting assays, cells were used $48 \mathrm{~h}$ after transfection.

\section{RNA isolation and qRT-PCR assays}

Total RNA was extracted from cultured cells using TRIzol reagent (Invitrogen, Carlsbad, CA, USA) according to the manufacturer's protocol and quantified with Nanodrop 2000 (Thermo Fisher Scientific, Waltham, MA, USA). First-strand cDNA was synthesized with the PrimeScript RT Reagent Kit (TaKaRa, Shiga, Japan). Quantitative real-time polymerase chain reaction (qRT-PCR) was performed with SYBR Green premix Ex Taq (TaKaRa) with $\beta$-actin as an internal control. For all qRT-PCR analyses, the relative expression levels were compared to $\beta$-actin, and the unit was defined as $1 / 100,000$ of $\beta$-actin level. The details of primers used for qRT-PCR are summarized in Additional file 1: Table S1.

\section{In vitro migration and invasion assays}

Cell migration and invasion assays were performed in 24-well plates with $8-\mathrm{mm}$ pore size chamber inserts (Corning, NY, USA), following established procedures. For migration assays, $5 \times 10^{4}$ cells were placed into each well of the upper chamber with the non-coated membrane. For invasion assays, $1 \times 10^{5}$ cells were placed into the upper chamber with the Matrigel-coated membrane, which was diluted with serum-free culture medium. In both assays, cells were suspended in $200 \mu \mathrm{l}$ of DMEM without fetal bovine serum when they were seeded into the upper chamber. In the lower chamber, $800 \mu \mathrm{l}$ of DMEM supplemented with $10 \%$ fetal bovine serum was added. After incubation for $16 \mathrm{~h}$ (migration assay) or $24 \mathrm{~h}$ (invasion assay) under normoxic or hypoxic conditions, the membrane inserts were removed from the plate, and non-invading cells were removed from the upper surface of the membrane. Cells that moved to the bottom surface of the chamber were fixed with $100 \%$ methanol for $20 \mathrm{~min}$ and stained with $0.1 \%$ crystal violet for $30 \mathrm{~min}$. Then, at least 10 randomly selected fields were imaged and the cell numbers counted under a CKX41 inverted microscope (Olympus, Tokyo, Japan). The assays were conducted with at least three independent repeats.

\section{Western blotting analysis}

Cell lysates were extracted from cultured cells with a mixture of T-PER Protein Extraction Reagent (Thermo Fisher Scientific), PhosSTOP (Roche, Basel, Switzerland) and Complete Mini (Roche). Protein samples were separated by $6 \%$ and $8 \%$ sodium dodecyl sulfate-polyacrylamide gel electrophoresis (SDS-PAGE) and transferred to nitrocellulose filter membranes (Millipore, Billerica, USA). After blocking in phosphate-buffered saline/Tween-20 containing $5 \%$ non-fat milk, the membranes were incubated with the following primary antibodies: DEC2 (Proteintech Group, Chicago, USA), HIF-1 $\alpha$ (Proteintech Group, Chicago, USA), $\beta$-actin (Sigma-Aldrich). Horse radish peroxidaseconjugated anti-rabbit IgG (Sigma-Aldrich) was used as the secondary antibody. Subsequent visualization was detected with SuperSignal West Femto Maximum Sensitivity Substrate (Thermo Fisher Scientific).

\section{Immunohistochemistry (IHC)}

Immunohistochemistry was performed with procedures similar to that described previously [29]. Briefly, formalinfixed and paraffin-embedded sections were stained for rabbit anti-human HIF-1 $\alpha$ polyclonal antibody (1:200; 20960-1-AP, Proteintech Group) and rabbit anti-human DEC2 polyclonal antibody (1:50; S8568, Sigma-Aldrich) using multi-use secondary antibody (1:1000; Dako, Ely, UK). Staining was visualized with the EnVision ${ }^{\mathrm{TM}}$ HRP Rabbit/Mouse detection kit (Dako). All experimental procedures were performed according to the IHC staining 
protocol of corresponding manufacturers. Image acquisition was performed using an Olympus BX40 microscope with a $20 \times$ or $40 \times$ objective. Staining intensity was evaluated by systematically screening all slices and evaluating them according to an established 0-3 scale. The staining results for HIF-1 $\alpha$ protein were classified as follows: 0 , no staining; 1 , nuclear staining in $<1 \%$ of cells; 2 , nuclear staining in $1-10 \%$ of cells and/or weak cytoplasmic staining; 3 , nuclear staining in $>10 \%$ of cells and/or distinct or strong cytoplasmic staining [30]. For DEC2 protein, the staining was evaluated with the same criteria. Samples graded as 0 and 1 were considered negative, and those graded as 2 and 3 were considered positive. Two triple-negative breast cancer (TNBC) lines known to have strong expression of DEC2 and HIF-1 $\alpha$ [22] were used as positive controls. For negative controls, primary antibodies were substituted by PBS.

\section{Statistical analysis}

All statistical analyses were performed using the Statistical Package for Social Sciences software (version 19.0) (SPSS, Inc., USA). Spearman's rank correlation was used to determine the correlation between DEC2 and HIF-1 $\alpha$. Kaplan-Meier survival curve method was applied in the comparison of the Disease-free Survival between groups of HIF- $1 \alpha$ positive and negative expressions. Data were plotted with GraphPad Prism 5 software (GraphPad Software, Inc, La Jolla, CA, USA). Quantitative variables were presented as means with standard error of mean (s.e.m.), unless otherwise noted, and analyzed by Student's $t$-test between two groups (two-tailed; $\mathrm{P}<0.05$ was considered statistically significant).

\section{Results}

\section{Expression of DEC2 and HIF-1a in human osteosarcoma} specimens correlates with poor prognosis

To investigate the involvement of HIF- $1 \alpha$ and DEC2 in the progression and metastasis of osteosarcoma, we used a cohort of 50 primary osteosarcoma samples obtained from clinical patients. Immunohistochemistry results showed that 29 cases were positive for HIF- $1 \alpha(58 \%)$ and 13 specimens were positive for DEC2 (26\%) (Figure 1A). Among these 50 cases, 24 cases of osteosarcoma were followed up successfully. Using Kaplan-Meier survival analysis (Figure 1B), we found that high expression of HIF- $1 \alpha$ was correlated with higher probability of metastasis and significantly reduced disease-free survival (DFS), suggesting that HIF-1 activation may be a determining factor for metastasis and EFS in osteosarcoma.

Since DEC2 has been reported as a regulator of HIF- $1 \alpha$ stability in breast and endometrial cancers [22,23], we next asked if DEC2 negatively correlates with HIF-1 activation in osteosarcoma. We surprisingly found that among the 13 DEC2 positive cases, 11 were also HIF- $1 \alpha$ positive.

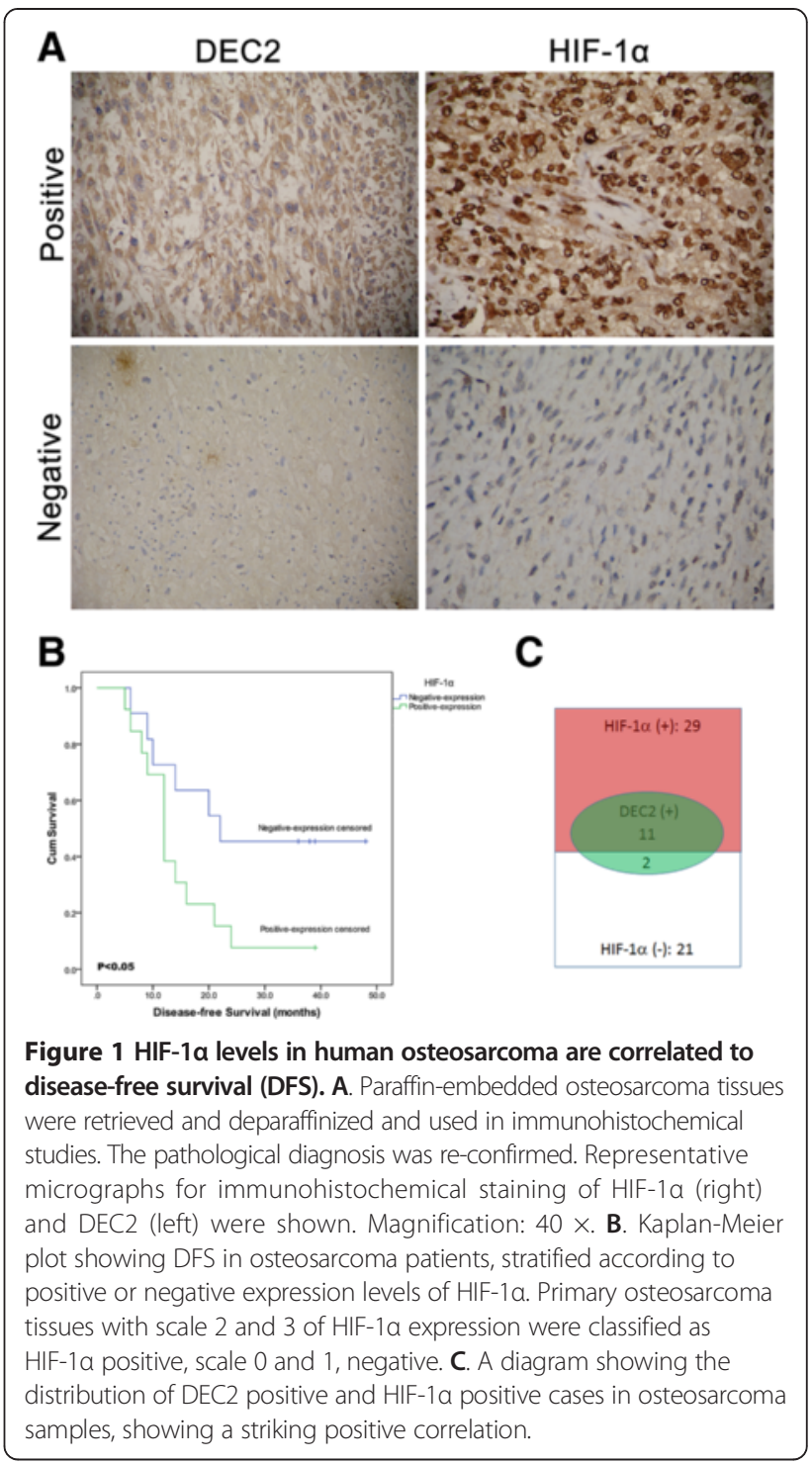

Statistical analysis revealed a strong positive correlation between DEC2 and HIF- $1 \alpha$ expression in osteosarcomas (Table 1, Spearman's rank correlation, ${ }^{*} \mathrm{P}<0.01$ ). These findings suggest a positive role of DEC2 in HIF-1 $\alpha$ stability in osteosarcoma, which may be different from that reported in other types of tumors.

\section{DEC2 facilitates HIF-1a expression in osteosarcoma cell lines}

To further investigate the functional interaction between DEC2 and HIF- $1 \alpha$, we synthesized siDEC2 and negative control siRNA (siControl). Using these siRNA, we knocked down the expression of DEC2 in osteosarcoma cell lines U2OS, MNNG and 143B, followed by exposing the cells to either normoxia or hypoxia. Western blotting analysis revealed that under normoxic conditions HIF- $1 \alpha$ was expressed in MNNG and 143B cells, but was not 
Table 1 Correlation between the expression of DEC2 and HIF-1a in human osteosarcomas

\begin{tabular}{llllll}
\hline DEC2 & \multicolumn{4}{l}{ HIF-1a expression scale* } & Total \\
\cline { 2 - 5 } & $\mathbf{0}$ & $\mathbf{1}$ & $\mathbf{2}$ & $\mathbf{3}$ & \\
\hline 0 & $0(0 \%)$ & $4(8 \%)$ & $2(4 \%)$ & $1(2 \%)$ & $7(14 \%)$ \\
1 & $2(4 \%)$ & $13(26 \%)$ & $11(22 \%)$ & $4(8 \%)$ & $30(60 \%)$ \\
2 & $0(0 \%)$ & $2(4 \%)$ & $2(4 \%)$ & $7(14 \%)$ & $11(22 \%)$ \\
3 & $0(0 \%)$ & $0(0 \%)$ & $0(0 \%)$ & $2(4 \%)$ & $2(4 \%)$ \\
Total & $2(4 \%)$ & $19(38 \%)$ & $15(30 \%)$ & $14(28 \%)$ & $50(100 \%)$ \\
\hline
\end{tabular}

Spearman's rank correlation, $\mathrm{P}<0.01, \mathrm{r}_{\mathrm{s}}=0.425$.

${ }^{*} 0-3$ scale represents the staining intensity as classified in the Materials and Methods.

detectable in U2OS cells (Figure 2). DEC2 knockdown did not affect HIF-1 $\alpha$ mRNA levels (Additional file 2: Figure S1 and data not shown). However, DEC2 knockdown effectively down-regulated HIF-1 $\alpha$ protein levels in MNNG and 143B cell lines under normoxic conditions (Figure 2), and in all three cell lines under hypoxic conditions, indicating that in osteosarcoma cells DEC2 positively regulates HIF- $1 \alpha$ at the post-transcriptional levels.

\section{DEC2 is required for optimal HIF-1a stabilization and HIF-1 activation upon hypoxia}

Under hypoxia or when driven by oncogenic signaling, HIF- $1 \alpha$ levels are stabilized to activate a set of target genes, such as angiopoietin-like 4 (ANGPTL4), vascular endothelial growth factor A (VEGFA) and hexokinase 2 (HK2), which promote adaptations at cellular, tissue and organismal levels $[6,31,32]$. In this study, we also found that hypoxia promoted U2OS cell invasiveness and migration (Additional file 3: Figure S2). We asked if DEC2 knockdown impairs hypoxia-stimulated HIF-1 $\alpha$ accumulation and HIF-1-dependent gene expression. We knocked down DEC2 expression in U2OS cells which have undetectable basal levels of HIF-1 $\alpha$ under normoxic condition, and cultured them under either normoxic or hypoxic condition for $48 \mathrm{~h}$. Using qRT-PCR, we confirmed that the siRNA efficiently knocked down DEC2 (Figure 3A, $\mathrm{P}>0.05$ ), and that DEC2 knockdown did not affect HIF- $1 \alpha$ mRNA levels (Figure 1). The mRNA levels of ANGPTL4, VEGFA, HK2 and PGK1, four classical HIF-1 downstream target genes, were stimulated by hypoxia in U2OS cells; however, DEC2 knockdown partially suppressed the hypoxia-stimulated expression of these HIF-1 target genes (Figures 3B-E). Similar results were obtained in MNNG and 143B cells (Figure 3F and Additional file 4: Figure S3). These results indicate a distinctive role of DEC2 in osteosarcoma, which facilitates HIF-1 activation and is apparently different from those observed in other types of tumors [22,23].

\section{Increased expression of DEC2 and HIF-1a is associated with increased invasiveness and metastatic potential of osteosarcomas}

To further explore the role of DEC2 and HIF- $1 \alpha$ in osteosarcoma progression, we used the repetitive trans-well approach $[27,33]$ to select a more invasive subpopulation from the parental U2OS cells. After a total of six rounds of selection, we obtained an invasive subpopulation named U2OS-M. As shown in Figure 4A and B, the migration and invasion ability of U2OS-M cells were significantly higher than that of the parental U2OS cells. We next asked if the increased invasiveness and migration potential are associated with HIF-1 activation and DEC2 expression. We found that the mRNA levels of HIF- $1 \alpha$ were not changed, but that the mRNA levels of DEC2 were significantly higher in U2OS-M than U2OS (Figure 4C). Consistent with the mRNA levels, the protein levels of DEC2 in U2OS-M cells were also remarkably higher than that in U2OS (Figure 4D, left part **: $\mathrm{P}<0.001)$. To investigate if increased expression of DEC2 affects the hypoxic stabilization of HIF-1 $\alpha$, we treated both U2OS and U2OS-M with hypoxia for $1 \mathrm{~h}$. Western blotting indicated that under normoxic conditions, neither U2OS nor U2OS-M had detectable HIF- $1 \alpha$ protein. However, after hypoxic treatment, U2OS-M cells accumulated HIF- $1 \alpha$ more rapidly than the parental U2OS cells (Figure 4D, right part). The role of DEC2 was further confirmed when DEC2 knockdown in U2OS-M cells impeded cell invasiveness and migration (Figure $4 \mathrm{E}$ and $\mathrm{F},{ }^{* * * *} \mathrm{P}<0.001$ ) and slowed down HIF-1 $\alpha$ accumulation (Figure 4G). These results indicate that

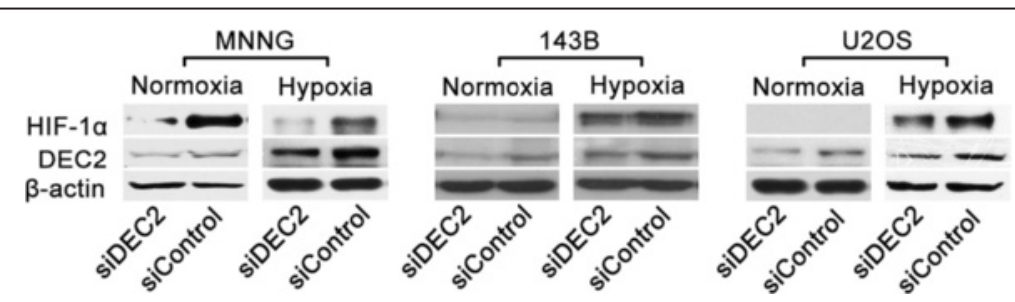

Figure 2 The effects of DEC2 knockdown on HIF-1a protein levels in osteosarcoma cell lines. DEC2 was knocked down in U2OS, MNNG and $143 \mathrm{~B}$ cell lines: Cells were transfected with indicated siRNAs and cultured for $48 \mathrm{~h}$ under either normoxia or hypoxia prior to cell harvest and protein sample collection. HIF-1a and DEC2 levels were determined by Western blotting, and $\beta$-actin was determined for normalization of sample loading. 

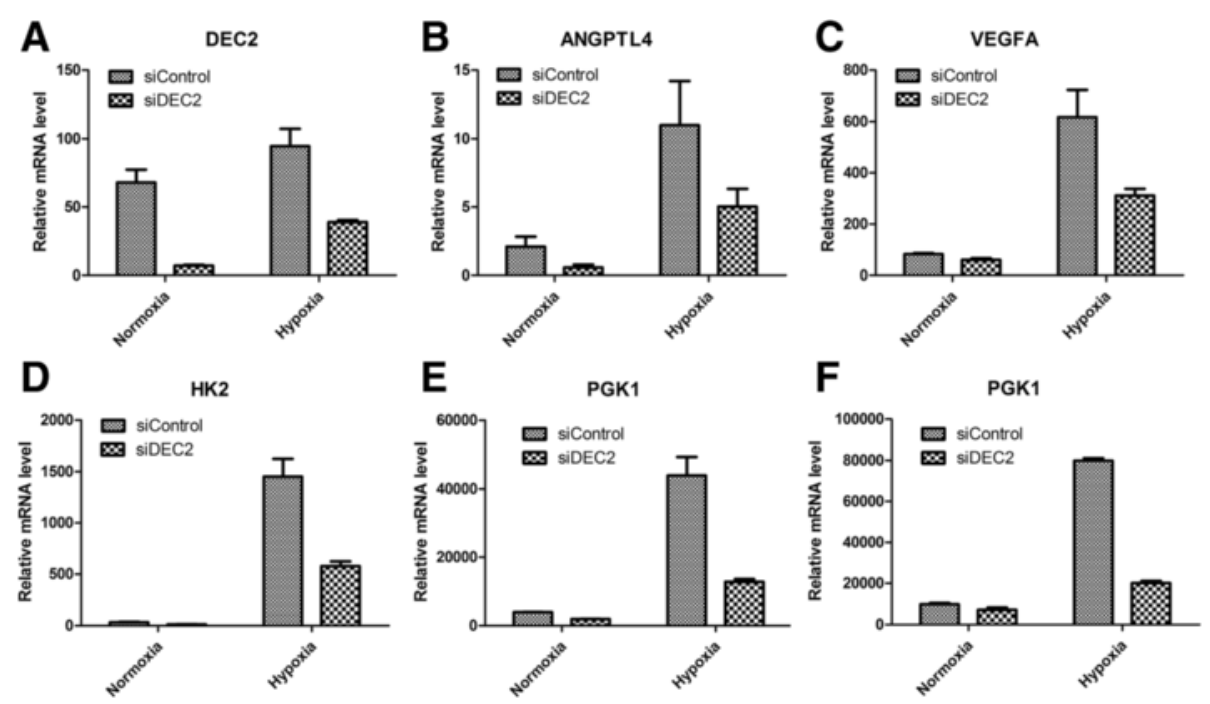

Figure 3 DEC2 is required for HIF-1a transactivation activity in U2OS. Cells were transfected with either control (NC) or DEC2 siRNAs and exposed to either $21 \%$ or $1 \%$ oxygen for $8 \mathrm{~h}$. A. Confirmation of DEC2 knockdown by qRT-PCR. B-F. Analyses of the expression levels of HIF-1a downstream target genes ANGPTL4 (B), VEGFA (C), HK2 (D), PGK1 (E) in U2OS cells and PGK1 (F) in MNNG cells by qRT-PCR.

DEC2 may contribute to the invasiveness of osteosarcoma by facilitating hypoxic HIF-1 $\alpha$ accumulation.

\section{HIF-1 activation is sufficient to upregulate DEC2 in osteosarcomas}

Finally, we asked how DEC2 expression is regulated in osteosarcomas. We performed a bioinformatics analysis of the DEC2 promoter, seeking transcription factors that could potentially interact with the DEC2 promoter. The 5,045 bp region encoding the DEC2 gene is located on the reverse strand Chromosome 12: 26,272,959-26,278,003 in GRCh37 coordinates. With the UCSC Genome Browser (http://genome.ucsc.edu/), the 2,000 bp upstream of the DEC2 transcription initiation site (chr12:26,278,804$26,280,003)$ was found to contain regions associated with highly acetylated histone $\mathrm{H} 3$ at lysine 27 (H3K27Ac) which indicates transcription regulator binding sites. Layered H3K27Ac is shown in Figure 5A, and Transcription Factor ChIP (Txn Factor ChIP) was chosen to evaluate the regulatory elements based on ENCODE Project (https://www.encodeproject.org/). Due to the limited number of transcription factors included in ENCODE Project, PROMO (http://alggen.lsi.upc.es/) was also utilized to predict transcription regulators of DEC2. From these analyses, binding sites for ninety seven transcription factors were predicted within a dissimilarity lesser than $5 \%$. Particularly, the analyses revealed that one H3K27Ac region contains putative sites for $\mathrm{c}-\mathrm{Fos} / \mathrm{Jun}$ and $\mathrm{c}-\mathrm{Myc} /$ Max (Figure 5A), two transcription factors encoded by oncogenes that are frequently activated in osteosarcomas [34]. This region also contains putative binding sites for E2F, a family of cell cycle regulatory transcription factors controlled by the retinoblastoma family of tumor suppressors ( $\mathrm{Rb})$ [35]. Interestingly, the analyses revealed three potential HIF-1 binding sites located at chr12:26,278,014-26,278,022 (TCCGCACGT), chr12:26,278,851-26,278,859 (AAAGCACGT) and chr12: 26,279,908-26,279,916 (ACGTGCCCT) (Figure 5A). A review of our qRT-PCR data revealed that hypoxia increased DEC2 mRNA levels (Figure 3A). To confirm whether HIF-1 activation is sufficient to upregulate DEC2, we treated 143B cells with desferrioxamine (DFX), a prolyl-hydroxylase inhibitor which stabilizes HIF-1 $\alpha$ under normoxic conditions [13]. As shown in Figure 5B, either hypoxia or DFX treatment was able to stabilize HIF-1 $\alpha$; the normoxic stabilization of HIF- $1 \alpha$ by DFX exposure effectively upregulated DEC2 expression, indicating HIF-1 activation is sufficient to enhance DEC2 expression in osteosarcomas.

\section{Discussion}

As the master regulator of glucose utilization and tissue angiogenesis, HIF-1 is activated in many solid tumors during tumor progression to sustain energy metabolism and biosynthesis [36], and to promote tumor cell migration [37]. In addition to hypoxia, oncogene activation and loss of tumor suppressor genes' function may contribute to normoxic activation of HIF-1 [13,34,35,38]. Glucose transporter 1 (GLUT1), HK2, ANGPTL4 and VEGF are well known downstream target genes of HIF-1 and are associated with tumor specific metabolic reprogramming, angiogenesis and metastasis. In this study, we demonstrated that HIF-1 $\alpha$ positivity predicts an unfavorable prognosis of osteosarcomas. Considering the previous reports that 


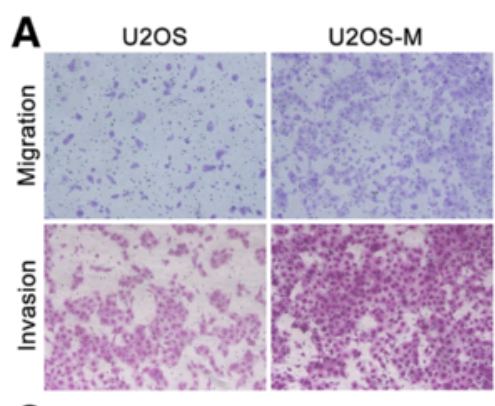

B

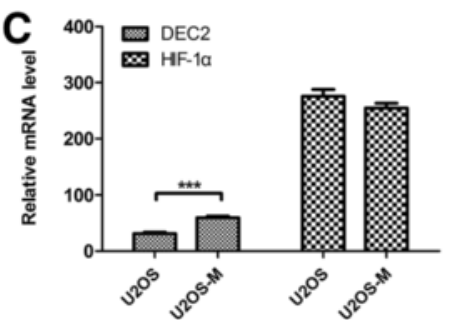

E

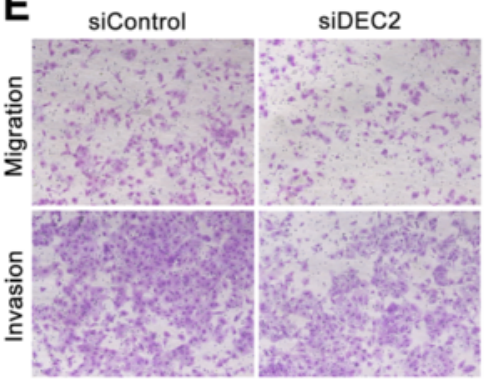

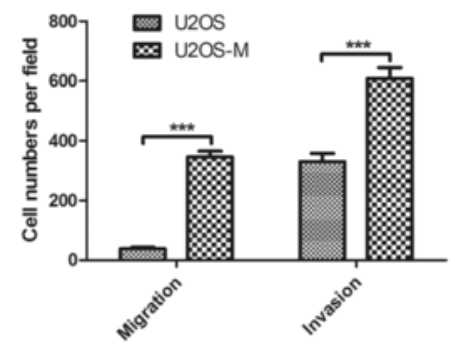

D

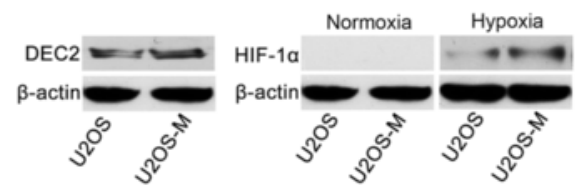

G

$\mathbf{F}$

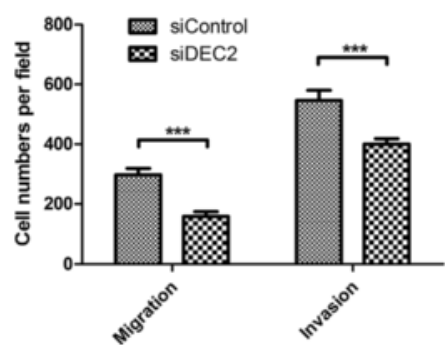

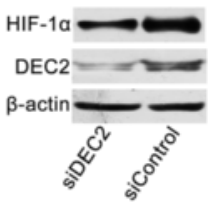

Figure 4 Invasive U2OS-M cells have higher levels of DEC2 and accumulate HIF-1a more rapidly in response to hypoxia. A. In vitro migration and invasion activity of U2OS and U2OS-M. Migration and invasion activities were measured in vitro with trans-well chambers, as described in Materials and Methods. Photos are representative fields of invasive cells on the membrane. Magnification: $100 \times$. B. Quantification of data shown in A. Bar graphs represent the average number of cells on the underside of the membrane (means \pm s.e.m.) C. U2OS-M cells express higher level of DEC2 mRNA than the parental U2OS, while the HIF-1 a mRNA level was not significantly affected as determined by qRT-PCR $(P>0.05)$. D. U2OS-M cells express higher level of DEC2 protein than U2OS cells. Cells were cultured under regular culture conditions or hypoxic condition (1 h) prior to harvest. Protein levels were determined by Western blotting. Under regular culture conditions, neither U2OS nor U2OS-M showed detectable HIF-1a. After $1 \mathrm{~h}$ hypoxic exposure, U2OS-M cells, which express higher level of DEC2, accumulated more HIF-1a than U2OS. E. U2OS-M Cells were transfected with either control or DEC2 siRNAs for $48 \mathrm{~h}$. Migration and invasion activities were measured in vitro with trans-well chambers, as described in Materials and Methods. Photos are representative fields of invasive cells on the membrane. Magnification: $100 \times$. F. Quantification of data shown in $\mathbf{E}$. Bar graph presents the average numbers of cells on the underside of the membrane (means \pm s.e.m.). G. DEC2 knockdown in U2OS-M slowed down HIF-1a accumulation. Cells were transfected with indicated siRNAs and cultured for $48 \mathrm{~h}$. Cells were exposed to hypoxia for $1 \mathrm{~h}$ prior to protein sample collection. HIF-1 $\mathrm{a}$ and DEC2 levels were determined by Western blotting, and $\beta$-actin was determined for normalization of loading. ${ }^{* *}: \mathrm{P}<0.001$.

DEC2 promoted HIF-1 $\alpha$ degradation in breast and endometrial cancers, one would expect a negative correlation between DEC2 and HIF-1 $\alpha$. Surprisingly, our clinical data based on studies on 50 osteosarcoma samples revealed that DEC2 expression levels have a positive relationship with HIF-1 $\alpha$ levels, raising the possibility that DEC2 overexpression predicts a poor prognosis of osteosarcoma, and may contribute to the development and progression of osteosarcomas. Indeed, we observed that HIF-1 target genes ANGPTL4, VEGFA, GLUT1, PGK1 and HK2 were down-regulated at mRNA level when DEC2 is knocked down in several osteosarcoma cell lines, while the mRNA level of HIF-1 $\alpha$ showed no obvious change by the DEC2 knockdown. Since HIF-1 $\alpha$ levels are mainly regulated at the level of protein stability [39], and HIF- $1 \alpha$ is remarkably down-regulated with the depletion of DEC2, our data suggest that DEC2 may be a facilitator for HIF-1 $\alpha$ stabilization in osteosarcomas under the influence of oncogenic signaling or in a hypoxic tumor microenvironment.

DEC2 is expressed in various embryonic and adult tissues, functioning as a transcription repressor of E-box activity $[18,19]$. Emerging evidence suggests that it suppresses tumor progression in oral, endometrial and breast cancers, and mechanistically, a role in apoptosis, proliferation and metastasis has been proposed [22,23,25]. 


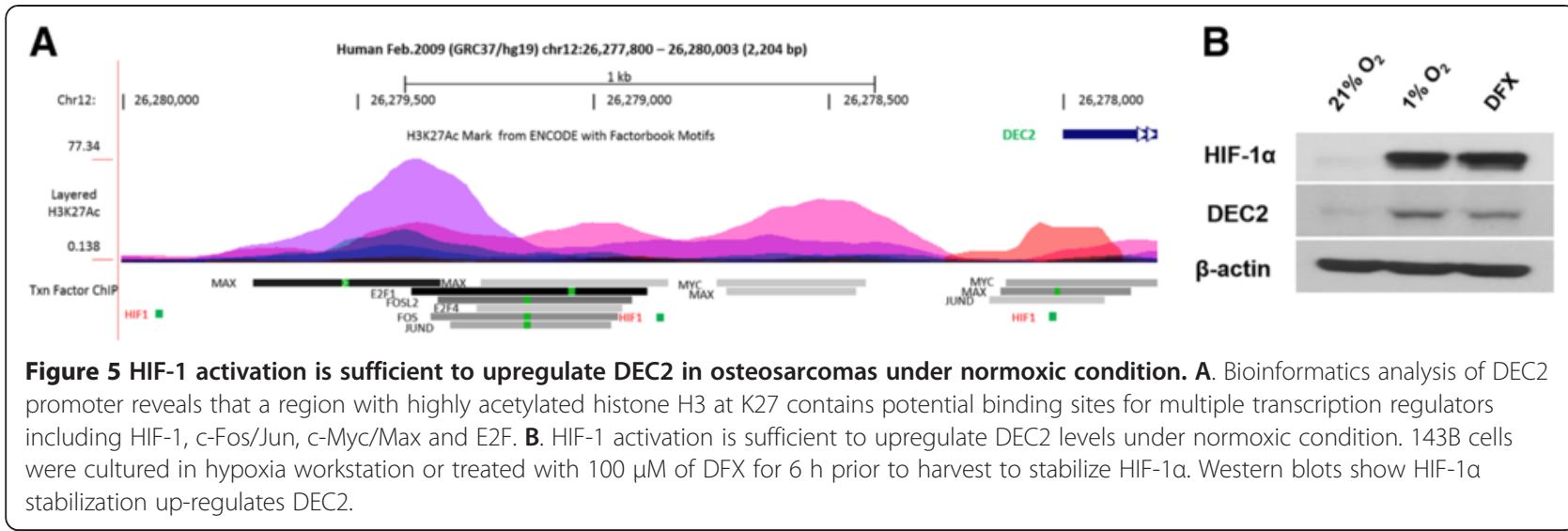

Generally, these previous studies suggested that DEC2 functions as a tumor suppressor. In this study, in 24 cases that were followed up to 3 years, high expression of HIF- $1 \alpha$ was shown to indicate a significantly higher probability of developing metastasis and of reduced DFS. Intriguingly, the expression level of DEC2 is positively correlated with HIF-1 $\alpha$. In addition, the expression of DEC2 indicates a tendency for poor prognosis. However, based on the current sample size, our available data failed to meet the criteria of statistics significance. A larger sample size is required to further examine the relationship between DEC2 expression and the prognosis of osteosarcomas.

Using an in vitro evolution model, we isolated a more invasive subpopulation (U2OS-M) from the human osteosarcoma U2OS cell line, which has been extensively used to investigate osteosarcoma metastasis $[27,28,40]$. Our data showed that the subpopulation selected from repetitive trans-well culture procedures has enhanced invading and metastasizing capabilities. Interestingly, compared with the parental U2OS cell line, the more invasive U2OS-M population has higher levels of DEC2. Whereas no HIF- $1 \alpha$ protein in either U2OS or U2OS-M cells under normal culture conditions was observed, U2OS-M cells accumulated HIF- $1 \alpha$ more rapidly than the parental U2OS cells after exposure to hypoxia, indicating the enhanced expression of DEC2 sensitizes osteosarcoma cells to hypoxia. The role of DEC2 in HIF-1 activation and invasiveness of osteosarcomas was further confirmed by DEC2 knockdown in U2OS-M lowered the fast hypoxic accumulation of HIF- $1 \alpha$ and the invasiveness.

Mechanistically, the reduction of HIF- $1 \alpha$ levels mediated by DEC2 knockdown was blocked in the presence of a proteasome inhibitor (data not shown), suggesting that lack of DEC2 function promotes the proteasomedependent degradation of HIF-1 $\alpha$. On the other hand, enhanced DEC2 may partially suppress the VHL-independent, ubiquitination-independent degradation pathway [41,42]. This is apparently the opposite of the observation from breast cancers, where DEC2 promoted VHL-independent degradation of HIF-1 $\alpha$. While the detailed mechanism underlying the DEC2-facilitated stabilization of HIF- $1 \alpha$ in osteosarcomas remains to be further explored, our results indicate that DEC2 could promote osteosarcoma invasiveness and metastasis by enhancing HIF-1 function.

While the transcriptional regulation of DEC2 expression may be complicated and affected by oncogenic signaling pathways (Figure 5A), we observed that DEC2 levels were enhanced by hypoxia in the osteosarcoma cell lines we studied. Importantly, stabilization of HIF- $1 \alpha$ is sufficient to enhance DEC2 protein levels, and inhibiting prolyl hydroxylase activity by DFX under normoxic condition also enhanced DEC2 levels (Figure 5B). Consistent with our observation, an early study reported that DEC2 promoter possesses a hypoxia responsive element between -863 and -258 bp (covering the predicted HIF-1 binding site at chr12:26,278,851-26,278,859), which physically bound HIF-1 in electrophoretic mobility shift assays [43]. Since multiple oncogenic signaling pathways may be activated thus triggering normoxic HIF-1 activation in osteosarcomas, it is very possible that the oncogenic activation of HIF-1 plays an important role in hypoxia-independent up-regulation of DEC2. It is also possible that the activation of c-Fos/Jun, c-Myc/Max or loss of $\mathrm{Rb}$ family tumor suppressors is able to trigger the up-regulation of DEC2, which facilitates HIF-1 activation upon hypoxia. As such, either case may provide an explanation for normoxic HIF-1 activation and DEC2 up-regulation in osteosarcoma. Further studies are needed to investigate these possibilities.

Taking together, we propose a HIF-1-DEC2 vicious cycle model in osteosarcoma (Figure 6). In this model, oncogenic signaling or spatiotemporal hypoxia in the osteosarcoma microenvironment stabilizes HIF-1 $\alpha$ and activates HIF-1. HIF-1 transcriptionally up-regulates DEC2, which in turn facilitates HIF-1 activation. This deregulated HIF-1 activation eventually contributes to the transcriptional reprograming, metabolic reprograming, angiogenesis and invasive nature of osteosarcomas. 


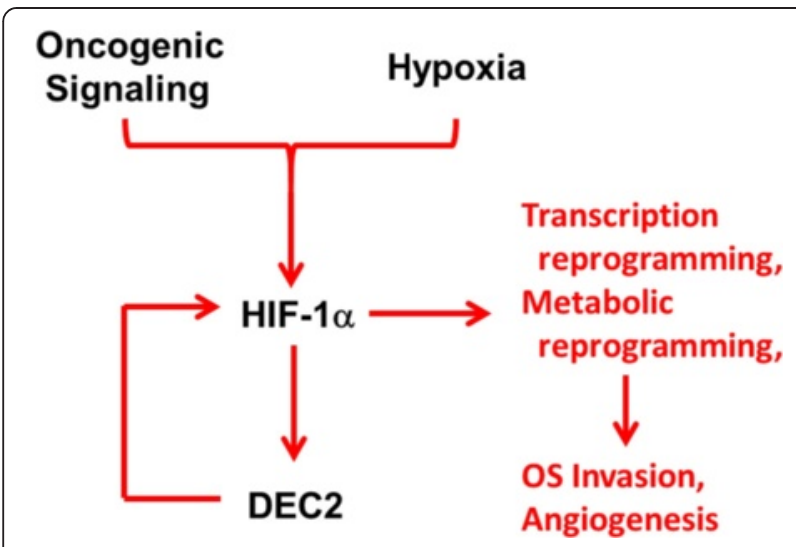

Figure 6 Proposed model showing the intriguing functional DEC2-HIF-1 association in the progression of osteosarcoma. In osteosarcomas, a combination of hypoxia and oncogenic signaling may increase the levels of HIF-1a, which upregulates DEC2 expression. DEC2 in turn facilitates the stabilization of HIF-1a, sensitizing osteosarcoma cells to hypoxic or oncogenic signaling and rapidly activating HIF-1 function.

As discussed above, DEC2 overexpression promotes HIF- $1 \alpha$ degradation in breast and endometrial cancers. It is particularly interesting, but remains unclear, why DEC2 functions differently in osteosarcomas. One possibility is that osteosarcomas have a dominant negative form of DEC2, which may have arosen by mutation, differential splicing or other mechanism. Since the molecular weight of DEC2 detected in osteosarcomas by Western blotting is very similar to that seen in other types of tumors, large deletion or truncation of DEC2 in osteosarcomas is unlikely. The mutation, if any, would be more likely to be in the form of a point mutation leading to an amino acid substitution. Whether osteosarcomas have a mutated form of DEC2 remains to be investigated by cloning and sequencing of the DEC2 gene from osteosarcomas, which is currently under way. It is also possible that DEC2 forms a protein complex that regulates HIF-1 $\alpha$ stability; stabilizing or destabilizing of HIF-1 $\alpha$ is determined by the association or dissociation with other components of the complex, or a change of subcellular localization regulated by the complex dynamics. Further studies are needed to decipher the molecular mechanisms underlying this intriguing mystery.

\section{Conclusion}

In contrast to other tumors, osteosarcoma shows that DEC2 expression positively correlates with HIF- $1 \alpha$ levels, and that high HIF-1 $\alpha$ expression levels predict poor prognosis. While DEC2 facilitates HIF- $1 \alpha$ stabilization, HIF-1 activation also upregulates DEC2 expression, forming a vicious cycle in osteosarcoma. The DEC2-HIF-1 vicious cycle may be triggered by either oncogenic signaling or the hypoxic microenvironment in osteosarcomas and so contribute to metabolic reprogramming and invasiveness. This provides a new prognostic biomarker and therapeutic target for highly invasive and metastatic osteosarcomas.

\section{Additional files}

Additional file 1: Table S1. Primers Used in GRT-PCR.

Additional file 2: Figure S1. Hypoxia enhances U2OS cell migration in vitro. Human U2OS osteosarcoma cells were exposed to either $21 \%$ or $1 \%$ oxygen. Cell migration and invasion were assessed as described in Materials and Methods. A. Representative photographs of migrated cells on the membrane at a magnification of $100 \times$. B. Data are presented as the means \pm s.e.m. of triplicate samples, and are representative of three independent experiments. ${ }^{*} \mathrm{P}<0.05$, ${ }^{*} \mathrm{P}<0.01$.

Additional file 3: Figure S2. DEC2 knockdown does not affect the mRNA levels of HIF-1a in U2OS under either normoxic or hypoxic conditions. Cells were transfected with either control (siControl) or DEC2 (siDEC2) siRNAs and exposed to either $21 \%$ or $1 \%$ oxygen levels for $8 \mathrm{~h}$. Total RNA were extracted, and the mRNA levels of HIF-1a were quantitated by qRT-PCR.

Additional file 4: Figure S3. DEC2 knockdown blunts the hypoxic stimulation of HIF-1 target genes GLUT1 and CAIX. Osteosarcoma cell line $143 \mathrm{~B}$ was transfected with control or DEC2 siRNAs and cultured for $48 \mathrm{~h}$. Then the cells were exposed to either $21 \%$ or $1 \%$ oxygen (In VIVO2, Ruskinn Technology Limited, UK) for $8 \mathrm{~h}$ and cells were harvested and total RNA extracted. The expression levels of GLUT1 and CAIX (CA9) were determined by quantitative RT-PCR with Taqman gene expression primers (Invitrogen) and StepOnePlus instrument (Applied Biosystems), and the mRNA levels were presented in relative quantity $(\mathrm{RQ})$.

\section{Abbreviations}

HIF-1: Hypoxia-inducible factor 1; DEC2: Differentiated embryonic chondrocyte gene 2; EFS: Event-free survival rate; ARNT1: Aryl hydrocarbon receptor nuclear translocator 1; MDM2: MDM2 proto-oncogene, E3 ubiquitin protein ligase; VEGFA: Vascular endothelial growth factor A; PGK1: Phosphoglycerate kinase 1; ANGPTL4: Angiopoietin-like 4; CAIX (CA9): Carbonic anhydrase IX; HK2: Hexose kinase 2; SHARP1: Enhancer-of-split and hairy-related protein 1; bHLHE41: basic helix-loop-helix family, member e41; $1 \mathrm{HC}$ : Immunohistochemistry; TNBC: Triple-negative breast cancer; DFS: Disease-free survival; H3K27Ac: Acetylated histone H3 at lysine 27; Txn Factor ChIP: Transcription Factor ChIP; DFX: Desferrioxamine;

GLUT1: Glucose transporter 1.

\section{Competing interests}

The authors declare that they have no competing interests.

\section{Authors' contributions}

$\mathrm{TH}, \mathrm{NH}$ carried out the DEC2 knockdown with U2OS and MNNG cells and drafted the initial version of manuscript. YY participated in the design of the study and performed the statistical analysis. CY contributed all experimental data from 143B cells, performed bioinformatics analyses and participated in manuscript drafting. QY conceived of the study, helped draft the manuscript and arranged collaboration among multiple groups, NS participated in experiment design, data interpretation, data organization and manuscript modification. All authors have read and approved the final manuscript.

\section{Authors' information}

Tu Hu and Nengbin He are co-first authors.

\section{Acknowledgements}

We are thankful to colleagues in the department of orthopedics for osteosarcoma sample collection. We are also grateful to Dr. M. Yao and his team in the Laboratory of Oncogenes and Related Genes, Shanghai Cancer Institute for their help and constructive discussion. We also thank Dr. M. King in Sydney Kimmel Cancer Center, Philadelphia for the kind gift of 143B cell line. Finally we thank Dr. S. Anandan (Drexel University) for helpful discussion and careful proofreading our manuscript prior to submission. This study is supported by the National Natural Science Foundation of China to QY (No. 30973017) and National Institute of Health, USA to NS (No. R01-CA129494). 


\section{Grant information}

Grant sponsor: National Natural Science Foundation of China; Grant number: 30973017.

Grant sponsor: National Institute of Health, USA; R01-CA129494.

\section{Author details}

'Department of Orthopedics, Shanghai Jiao Tong University Affiliated Sixth People's Hospital, No.600, Yishan Road, Shanghai 200233, China. ${ }^{2}$ Huazhong University of Science and Technology, Wuhan, China. ${ }^{3}$ Departments of Biology, Pathology \& Laboratory Medicine, Drexel University CoAS, 3245 Chestnut St, PISB 417, Philadelphia, PA 19104, USA. ${ }^{4}$ Kimmel Cancer Center, Thomas Jefferson University, Philadelphia, PA, USA.

Received: 15 January 2015 Accepted: 12 February 2015

\section{Published online: 28 February 2015}

\section{References}

1. Kubota D, Mukaihara K, Yoshida A, Tsuda H, Kawai A, Kondo T. Proteomics study of open biopsy samples identifies peroxiredoxin 2 as a predictive biomarker of response to induction chemotherapy in osteosarcoma. J Proteomics. 2013;91:393-404.

2. He JP, Hao Y, Wang XL, Yang XJ, Shao JF, Guo FJ, et al. Review of the molecular pathogenesis of osteosarcoma. Asian Pac J Canc Prev. 2014;15(15):5967-76

3. Mialou V, Philip T, Kalifa C, Perol D, Gentet JC, Marec-Berard P, et al. Metastatic osteosarcoma at diagnosis: prognostic factors and long-term outcome-the French pediatric experience. Cancer. 2005;104(5):1100-9.

4. Fu HL, Shao L, Wang Q, Jia T, Li M, Yang DP. A systematic review of p53 as a biomarker of survival in patients with osteosarcoma. Tumour Biol. 2013;34(6):3817-21.

5. Wu Q, Yang SH, Ye SN, Wang RY. Therapeutic effects of RNA interference targeting HIF-1alpha gene on human osteosarcoma]. Chin J Med [Zhonghua Yi Xue Za Zhi]. 2005;85(6):409-13.

6. Chen S, Sang N. Histone deacetylase inhibitors: the epigenetic therapeutics that repress hypoxia-inducible factors. J Biomed Biotechnol. 2011;2011:197946.

7. Cheng DD, Zhao HG, Yang YS, Hu T, Yang QC. GSK3beta negatively regulates HIF1alpha mRNA stability via nucleolin in the MG63 osteosarcoma cell line. Biochem Bioph Res Co. 2014;443(2):598-603.

8. Hirota K, Semenza GL. Regulation of angiogenesis by hypoxia-inducible factor 1. Crit Rew Oncol Hematol. 2006;59(1):15-26.

9. Sang N, Fang J, Srinivas V, Leshchinsky I, Caro J. Carboxyl-terminal transactivation activity of hypoxia-inducible factor 1 alpha is governed by a von Hippel-Lindau protein-independent, hydroxylation-regulated association with p300/CBP. Mol Cell Biol. 2002;22(9):2984-92.

10. Stiehl DP, Fath DM, Liang D, Jiang Y, Sang N. Histone deacetylase inhibitors synergize p300 autoacetylation that regulates its transactivation activity and complex formation. Cancer Res. 2007;67(5):2256-64.

11. Joshi S, Singh AR, Durden DL. MDM2 regulates hypoxic hypoxia-inducible factor 1alpha stability in an E3 ligase, proteasome, and PTENphosphatidylinositol 3-kinase-AKT-dependent manner. J Biol Chem. 2014;289(33):22785-97.

12. Joshi S, Singh AR, Durden DL. Pan-PI-3 kinase inhibitor SF1126 shows antitumor and antiangiogenic activity in renal cell carcinoma. Cancer Chemoth Pharm 2015 Epub ahead of print.

13. Sang N, Stiehl DP, Bohensky J, Leshchinsky I, Srinivas V, Caro J. MAPK signaling up-regulates the activity of hypoxia-inducible factors by its effects on p300. J Biol Chem. 2003;278(16):14013-9.

14. Amelio I, Melino G. The "Sharp" blade against HIF-mediated metastasis. Cell cycle (Georgetown, Tex). 2012;11(24):4530-5.

15. Figlin RA, de Souza P, McDermott D, Dutcher JP, Berkenblit A, Thiele A, et al. Analysis of PTEN and HIF-1alpha and correlation with efficacy in patients with advanced renal cell carcinoma treated with temsirolimus versus interferon-alpha. Cancer. 2009;115(16):3651-60.

16. Generali D, Buffa FM, Berruti A, Brizzi MP, Campo L, Bonardi S, et al. Phosphorylated ERalpha, HIF-1a, and MAPK signaling as predictors of primary endocrine treatment response and resistance in patients with breast cancer. J Clin Oncol. 2009;27(2):227-34.

17. Yang QC, Zeng BF, Dong Y, Shi ZM, Jiang ZM, Huang J. Overexpression of hypoxia-inducible factor-1alpha in human osteosarcoma: correlation with clinicopathological parameters and survival outcome. Jpn J Clin Oncol. 2007;37(2):127-34

18. Azmi S, Sun H, Ozog A, Taneja R. mSharp-1/DEC2, a basic helix-loop-helix protein functions as a transcriptional repressor of $\mathrm{E}$ box activity and Stra13 expression. J Biol Chem. 2003;278(22):20098-109.

19. Azmi $\mathrm{S}$, Taneja R. Embryonic expression of $\mathrm{mSharp}-1 / \mathrm{mDEC} 2$, which encodes a basic helix-loop-helix transcription factor. Mech Develop. 2002;114(1-2):181-5.

20. Li Y, Shen Q, Kim HT, Bissonnette RP, Lamph WW, Yan B, et al. The rexinoid bexarotene represses cyclin D1 transcription by inducing the DEC2 transcriptional repressor. Breast Cancer Res TR. 2011;128(3):667-77.

21. Liu Y, Sato F, Kawamoto T, Fujimoto K, Morohashi S, Akasaka H, et al. Anti-apoptotic effect of the basic helix-loop-helix (bHLH) transcription factor DEC2 in human breast cancer cells. Genes Cells. 2010;15(4):315-25.

22. Montagner M, Enzo E, Forcato M, Zanconato F, Parenti A, Rampazzo E, et al. SHARP1 suppresses breast cancer metastasis by promoting degradation of hypoxia-inducible factors. Nature. 2012;487(7407):380-4.

23. Liao Y, Lu W, Che Q, Yang T, Qiu H, Zhang H, et al. SHARP1 suppresses angiogenesis of endometrial cancer by decreasing hypoxia-inducible factor-1alpha level. PLoS ONE. 2014;9(6):e99907.

24. Sato F, Bhawal UK, Kawamoto T, Fujimoto K, Imaizumi T, Imanaka T, et al Basic-helix-loop-helix (bHLH) transcription factor DEC2 negatively regulates vascular endothelial growth factor expression. Genes Cells. 2008;13(2):131-44.

25. Wu Y, Sato F, Bhawal UK, Kawamoto T, Fujimoto K, Noshiro M, et al. BHLH transcription factor DEC2 regulates pro-apoptotic factor Bim in human ora cancer HSC-3 cells. Biomed Res (Tokyo, Japan). 2012;33(2):75-82.

26. King MP, Attardi G. Human cells lacking mtDNA: repopulation with exogenous mitochondria by complementation. Science. 1989:246(4929):500-3.

27. Tie J, Pan Y, Zhao L, Wu K, Liu J, Sun S, et al. MiR-218 inhibits invasion and metastasis of gastric cancer by targeting the Robo1 receptor. PLoS Genet. 2010;6(3):e1000879.

28. Torng PL, Lee YC, Huang CY, Ye JH, Lin YS, Chu YW, et al. Insulin-like growth factor binding protein-3 (IGFBP-3) acts as an invasion-metastasis suppressor in ovarian endometrioid carcinoma. Oncogene. 2008;27(15):2137-47

29. Lin S, Guo Q, Wen J, Li C, Lin J, Cui X, et al. Survival analyses correlate stanniocalcin 2 overexpression to poor prognosis of nasopharyngeal carcinomas. J Exp Clin Canc Res. 2014;33:26.

30. Ishikawa T, Nakashiro K, Klosek SK, Goda H, Hara S, Uchida D, et al. Hypoxia enhances CXCR4 expression by activating HIF-1 in oral squamous cell carcinoma. Oncol Rep. 2009;21(3):707-12.

31. Minchenko A, Leshchinsky I, Opentanova I, Sang N, Srinivas V Armstead $V$, et al. Hypoxia-inducible factor-1-mediated expression of the 6-phosphofructo-2-kinase/fructose-2,6-bisphosphatase-3 (PFKFB3) gene. Its possible role in the Warburg effect. J Biol Chem. 2002;277(8):6183-7.

32. Zhang $T$, Niu X, Liao L, Cho EA, Yang H. The contributions of HIF-target genes to tumor growth in RCC. PLOS ONE. 2013;8(11):e80544.

33. Lu KV, Jong KA, Rajasekaran AK, Cloughesy TF, Mischel PS. Upregulation of tissue inhibitor of metalloproteinases (TIMP)-2 promotes matrix metalloproteinase (MMP)-2 activation and cell invasion in a human glioblastoma cell line. Lab Invest. 2004;84(1):8-20.

34. Tang N, Song WX, Luo J, Haydon RC, He TC. Osteosarcoma development and stem cell differentiation. Clin Orthop Relat R. 2008;466(9):2114-30.

35. MacLachlan TK, Sang N, Giordano A. Cyclins, cyclin-dependent kinases and cdk inhibitors: implications in cell cycle control and cancer. Crit Rev Eukar Gene. 1995:5(2):127-56.

36. Yin C, Qie S, Sang N. Carbon source metabolism and its regulation in cancer cells. Crit Rev Eukar Gene. 2012;22(1):17-35.

37. Manalo DJ, Rowan A, Lavoie T, Natarajan L, Kelly BD, Ye SQ, et al. Transcriptional regulation of vascular endothelial cell responses to hypoxia by HIF-1. Blood. 2005;105(2):659-69.

38. Hu X, Yu AX, Qi BW, Fu T, Wu G, Zhou M, et al. The expression and significance of IDH1 and p53 in osteosarcoma. J Exp Clin Canc Res. 2010;29:43.

39. Kaelin Jr WG, Ratcliffe PJ. Oxygen sensing by metazoans: the central role of the HIF hydroxylase pathway. Mol Cell. 2008;30(4):393-402.

40. Chu YW, Yang PC, Yang SC, Shyu YC, Hendrix MJ, Wu R, et al. Selection of invasive and metastatic subpopulations from a human lung adenocarcinoma cell line. Am J Resp Cell Mol. 1997;17(3):353-60. 
41. Kong X, Lin Z, Liang D, Fath D, Sang N, Caro J. Histone deacetylase inhibitors induce $\mathrm{VHL}$ and ubiquitin-independent proteasomal degradation of hypoxia-inducible factor 1a. Mol Cell Biol. 2006;26(6):2019-28

42. Lao T, Chen S, Sang N. Two mutations impair the stability and function of ubiquitin-activating enzyme (E1). J Cell Physiol. 2012;227(4):1561-8.

43. Miyazaki K, Kawamoto T, Tanimoto K, Nishiyama M, Honda H, Kato Y. Identification of functional hypoxia response elements in the promoter region of the DEC1 and DEC2 genes. J Biol Chem. 2002;277(49):47014-21.

Submit your next manuscript to BioMed Central and take full advantage of:

- Convenient online submission

- Thorough peer review

- No space constraints or color figure charges

- Immediate publication on acceptance

- Inclusion in PubMed, CAS, Scopus and Google Scholar

- Research which is freely available for redistribution 